\title{
Some Results for a Time Interval Approach to Field Theory and Gravitation
}

\author{
Harmen H. Hollestelle \\ Independent researcher, Common Room/Networks Foundation, Bandung/Sukabumi 40191, West-Java, \\ Indonesia \\ harmhollestelle@yahoo.com
}

\begin{abstract}
This paper consists of two parts. In part I some new relations for a field theory with time intervals are derived. One concept of field theory evaluated is complementarity, another is field operators both defined within a time interval description. Part II includes specific results and commentary. Discussed are time interval dependent wave propagation surfaces for star source emission waves and derived is a metric propagation surface area requirement. The results allow to consider one same field that like gravitation within General Relativity applies to both non zero and zero mass. The associated field energy is space time dependent for non zero mass, and is related to a space time dependent metric tensor for zero mass wave particles. Defined is internal energy transfer where wave particle numbers increase linearly and mass and momentum diminish, decrease inversely with the distance from the wave emission source. The commentary are applications related to cosmological overall volume and temperature dependence.
\end{abstract}

Keywords: Time interval, equilibrium, field theory, complementarity, field operator, gravitation, wave propagation surface, emission source, cosmology

\section{Introduction: The time interval description and asymmetry of time coordinates}

The occurrence of an event in space time can be defined with coordinate domains. Newtonian mechanics depends on vectors that can be added giving the concept of velocity a meaning. Euclidean n-space with the vector addition property is a vector space over the field of real numbers. Because of the application of one value coordinate relations in space time to describe gravitational interaction by Newton, and by Minkowski and Einstein, the description with time moments, that is, one moment time, became the standard description of events, [Hocking, Young, 1], [Einstein, 2].

Specific for the time interval description is the approach towards change and time dependence.

Equilibrium is maintained by the application of the "mean velocity theorem" (MV) that allows time intervals to be asymmetric, ie different from the symmetric situation with a time independent Hamiltonian. In particular derivatives to time intervals instead of one moment time derivatives are included. The time interval description follows the definitions in [Hollestelle, 3].

The discussion of coordinate domains where quantum mechanics (qm) is or is not valid concentrates on the experimentation language needed to relate experimental results to theories [Roos, 4]. Within the experimentation coordinate domain one can describe phenomena like matter by introducing the concept of center of weight coordinates. This corresponds with the standard space time description, the time moment description. When the qm wave function after measurement is transformed or changed, which can be confirmed by a second successive measurement, this transformation is known as wave function reduction [Roos, 5]. 
With the introduction of time intervals and MV, comparative, equilibrium it is possible to describe Hamiltonian $\mathrm{H}$ time independent situations with the same results as the time moment description with Lagrangian equilibrium. When the Hamiltonian is time dependent still time intervals and MV equilibrium can be applied however with results different from time moment results, because of the difference maintaining equilibrium.

A basic concept of quantum mechanics is the occurrence of events with change. The photo-electric event that includes wave function collapse is one example. Events with change in the sense of wave function collapse can occur when the Hamiltonian is time dependent. The application of time intervals allows a clear and consistent description in these cases.

The time interval description depends on three concepts: time intervals and derivatives to time intervals, instead of one moment time derivatives, and MV comparative equilibrium instead of Lagrangian equilibrium.

The description and interpretation of time intervals and time coordinates follows the definitions from [ref. 3]. Any time interval $\Delta \mathrm{t}=[\mathrm{tb}, \mathrm{ta}]$ is defined such that one moment time coordinate ta is part of the future while tb is part of the past. A time interval is a unit and tb and ta are not independent and the indication past or future for them is part of this unit when remaining within the time interval description. One can describe this interdependence for instance with $\mathrm{tb}=\mathrm{tb}(\mathrm{ta})$ or alternatively $\mathrm{ta}=\mathrm{ta}(\mathrm{tb})$ while the time order for tb and ta remains the same. The domain for time intervals with time coordinates tb and ta, includes dependence on a "anti-symmetrical" element, this element being the same for a past tb and future ta, and dependence on "symmetrical" elements, being not necessarily opposite, for these same tb and ta. In this way past and future are regarded to have "similar" properties and "opposite" ones. The elements for tb and ta are the basis for the interdependence of tb and ta. Together they define a time interval and its unity. $|\Delta \mathrm{t}|$ is not so much an uncertainty rather a variation. With $\Delta \mathrm{t}$ can be described real existing events, however not defined are time intervals only in the future or only in the past.

A time interval derivative of a quantity or entity A to time interval $\Delta \mathrm{t}$ is indicated with " $\mathrm{dA} / \mathrm{dt}$ " $\| \Delta \mathrm{t}$ which depends on the complete time interval $\Delta \mathrm{t}$ while the one moment time derivative of $\mathrm{A}$ in the time moment description is indicated with $\mathrm{dA} / \mathrm{dt} \mid \mathrm{t}$ meaning its value at a specific one moment time $\mathrm{t}$. The definition of time interval derivatives follows in paragraph 5. "Comparative" equilibrium for time interval events is, instead of with Lagrangian equilibrium, introduced with the "mean velocity theorem" (MV theorem). The term comparative refers to asymmetry of properties, and is related to MV equilibrium during time interval $\Delta \mathrm{t}$, ie an interval [tb, ta] with time coordinate boundaries tb and ta, that includes asymmetry and time order and does not allow time reversal, paragraph 5. Time interval derivatives and MV equilibrium are defined and discussed extensively in [ref. 3].

This paper consists of the following parts. Part I. Field theory concepts and time intervals. Complementarity is reviewed within the time interval description in paragraph 2. and 3. Other traditional field theory concepts, observables, field limit process, and operators, are reviewed in paragraph 4 . A new definition for operators for field theory applying time intervals is proposed that depends on a time interval operator commutator, a "comparative" commutator, defined in paragraph 5, different from Lie or Poisson commutators and independent of the constant of Planck.

Part II. Discussion and comments. Within the time interval description it is derived field wave energy can vary in terms of a variable mass for the associated wave particle, paragraph 7 . To describe wave 
propagation from star like sources propagation surfaces are introduced where the surface wave energy Es is equal to a surface field energy Ee. The surface field depends on a specific surface curvature energy linear in the surface radius, defined in [Hollestelle, 6]. The equivalence depends on the field limit process for the construction of Ee and a metric area requirement for the wave propagation surface to be similar, paragraph 7.

Discussed is the emergence of a gravitation like inverse distance-energy relation derived for propagation surface field energy at varying distance, paragraph 7. This field energy, like with General Relativity (GR) and unlike Newtonian gravitation, associates with both non zero mass and zero mass wave particles.

Because of internal energy transfer, for non zero mass matter waves an inverse source-distance mass and momentum dependence occurs, where both properties diminish, corresponding with a linear with sourcedistance increasing wave particle number.

Several cosmological estimates related to overall volume and temperature are derived, paragraph 8 and 9. One of these includes how waves emitted by different sources, star like and localizable or cloud like and non localizable, are characterized by the wave emission source-distance.

\section{The time interval description, and wave momentum and complementarity}

Some of the fundamental relations of field theory depend on constants that occur or are defined by specific situations. When introducing time intervals and comparative equilibrium one has to re-evaluate these relations whether they can remain valid as fundamental. When some of the original relations are found to remain valid if even in altered form, related concepts can still be maintained, like complementarity. With derivatives to time intervals evaluating constants changes, and it can be necessary to re-write the relevant relation to include a variable. Applied are variables, instead of the constant of Planck h, to find time interval description complementarity energy relations while the complementarity relation for particle $\mathrm{p}$ and wave momentum $\mathrm{k}_{-}, \mathrm{p}=\mathrm{h} . \mathrm{k}_{-}$, is maintained.

The relation $\mathrm{E}=\mathrm{h} . v$ equates radiation wave energy with wave frequency $v$ through $\mathrm{h}$, the constant of Planck. The dot "." indicates ordinary numerical multiplication. This relation describes the energy unit for waves with frequency $v$, and is confirmed by photo-electric effect measurements [Sredniawa, 7], [Millikan, 8]. Such events include wave function collapse and a time dependent Hamiltonian H. Relation $\mathrm{E}=$ h.v refers to zero mass, ie radiation, waves, however some authors include non zero mass wave situations. It is assumed generally valid for radiation waves, whether time dependent or time independent $\mathrm{H}$, within the time moment description and for Lagrangian equilibrium. In [ref. 3] two time interval description functions related to $\mathrm{h}$ are defined, $\mathrm{h}+=1 / 2(\Delta \mathrm{p} \cdot \Delta \mathrm{q}+\Delta \mathrm{q} \cdot \Delta \mathrm{p})$ and $\mathrm{h}_{-}=(\Delta \mathrm{p} \cdot \Delta \mathrm{q}-\Delta \mathrm{q} \cdot \Delta \mathrm{p})$ where $\Delta$ is a variation related to time interval $\Delta \mathrm{t}$, while only in the $\mathrm{H}$ time dependent situation $\mathrm{h}+\neq \mathrm{h}$ and $\mathrm{h} \_\neq 0$. These two functions help to newly evaluate the above equation and others.

Now considered is the time interval description while $\mathrm{H}$ is allowed to be time dependent. Then, for situations where $h+$ not substantially differs from $h$, one could write the variable $h+$ instead of $h$ within the above equation as a transformation of the constant $h$. This approach is motivated by the fact that from the approximation $\mid \mathrm{h} \_$| "nearly however not completely" zero it follows, rather than $\Delta \mathrm{p} . \Delta \mathrm{q}=\mathrm{h}$, a constant, valid is $\Delta \mathrm{p} . \Delta \mathrm{q}=\mathrm{h}+$, a variable. A transformation of the constant $\mathrm{h}$ towards a variable, $\mathrm{h}+$ or another variable function h', is now applied as a general alternative for $\mathrm{H}$ time dependent events. A consequence when writing $\mathrm{E}=\mathrm{h}+. v$ is that, for $\Delta \mathrm{t}$ the relevant event time interval, $v$ is proportional to $1 /|\Delta \mathrm{t}|$ which is an 
unexpected relation that however not easily agrees with the finding in [ref. 3] that $1 /|\Delta t|$ decreases to zero for events towards the limit of $\mathrm{H}$ time independent, ie within the above approximation for $\left|\mathrm{h} \_\right| . \mathrm{E} \sim \mathrm{h}+/ \Delta \mathrm{t}$ is a relation derived in [ref. 3], one of the properties for radiation waves discussed below in paragraph 5. Because of this problematic result for an approach that includes a transformation of constant $h$ to a variable $\mathrm{h}+$ for the relation $\mathrm{E}=\mathrm{h} . \mathrm{v}$, the time moment description relation $\mathrm{E}=\mathrm{h} . v$ is regarded to be valid for all zero mass particle events including those with $\mathrm{H}$ time dependent, and justified also within the time interval description regardless the value of $\left|\mathrm{h} \_\right|$and regardless $\mathrm{H}$ time independent or time dependent.

Similarly, for non zero mass $\mathrm{m}$ waves the relation $\mathrm{E}=\mathrm{h} . \omega$ is regarded valid for all events in the time moment description, for both $\mathrm{H}$ time dependent or time independent. This is to be expected from the similarity of wave particle complementarity whether for zero mass or non zero mass. A derivation for time independent events of this relation within qm and the time moment description includes an average wave that is considered to be a composition of free waves, with a group velocity equal to the velocity of the associated particle. The weight-function $\mathrm{w}(\mathrm{k})$ with $\mathrm{k}$ a free wave momentum is assumed to be a block shape Heaviside function around the average momentum $\mathrm{k}_{-}$and limited by the k-domain for the occurrence of the momenta $\mathrm{k}$ that equals the block width [Merzbacher, 9].

For time dependent events a different block shape Heaviside like weight-function $w(k)$ is now assumed where the block shape is linear in $\mathrm{k}$ and asymmetric with a constant slope around a, the center of the kdomain. The average momentum $\mathrm{k}_{-}$and the center a of the $\mathrm{k}$-domain are different because of the asymmetry. Assume the time dependence of $\mathrm{k}(\mathrm{t})$ is similarly asymmetric during the event time interval $\Delta \mathrm{t}$ : $\mathrm{w}(\mathrm{k})(\mathrm{t})=\mathrm{w}(\mathrm{k}(\mathrm{t}))$ and any time development of $\mathrm{k}(\mathrm{t})$ and $\mathrm{w}(\mathrm{k})(\mathrm{t})$ results in asymmetry for $\mathrm{w}(\mathrm{k})$ that can be approximated with an asymmetric and linear slope block shape Heaviside k dependence. The proof of the validness of the time development relation for $\mathrm{w}(\mathrm{k})$ is indicated in the next paragraph 3 . It follows that in the time moment description $\mathrm{E}=\mathrm{h} . \omega$ remains valid for all non zero mass wave particle events, for $\mathrm{H}$ time independent and for $\mathrm{H}$ time dependent. Because the event time interval $\Delta \mathrm{t}$ occurs in the above argument in the same way for the time moment description and for the time interval description, this justifies that for all these non zero mass situations $\mathrm{E}=\mathrm{h} . \omega$ is regarded valid also within the time interval description.

\section{Time intervals, wave momentum, and asymmetry}

To introduce a time dependent $\mathrm{w}(\mathrm{k})$ the time moment description of the time independent situation is recalled. Left out are most numerical factors. Relation $\mathrm{E}=\mathrm{h} . \omega$ for non zero mass m matter waves, within a time independent situation, depends on the description of the "average" free wave exp(iq $\left.k_{-}-i \omega t\right)$ as the composition of free waves with a group velocity v_group that equals the constant velocity of the associated particle like in paragraph 2. When applying wave energy E equals Ekin the kinetic energy at time moment $\mathrm{t}$ and also $\mathrm{d} \omega / \mathrm{dk}_{-} \mid \mathrm{k}_{-}=\mathrm{v}_{-}$group, one finds $\omega\left(\mathrm{k}_{-}\right)=\mathrm{p} / \mathrm{m} . \mathrm{k}_{-}$and $\mathrm{E}=\mathrm{h} . \omega\left(\mathrm{k}_{-}\right)$. With $\mathrm{p}=\mathrm{h} . \mathrm{k}_{-}$ there is Ekin $=\left(h . k_{-}\right)^{\wedge} 2 / m$.

The weight-function $\mathrm{w}(\mathrm{k})$ for the free wave momentum $\mathrm{k}$ at any time moment can be approximated with: $\mathrm{w}(\mathrm{k})=\mathrm{w} 0$. Heaviside $(|\mathrm{k}-\mathrm{a}|-\mathrm{u})$ where $\mathrm{a}$ is the center of the $\mathrm{k}$-domain variance, where $\mathrm{a}=\mathrm{k}$ - for $\mathrm{H}$ time independent, and $\mathrm{u}$ is the measure of the variance where $|\mathrm{k}-\mathrm{a}|<|\mathrm{u}|$ and $|\mathrm{u}| \approx\left|\Delta^{\prime} \mathrm{k}\right|$. The $\Delta^{\prime}$ is a variance or uncertainty. When $\left|\Delta^{\prime} \mathrm{q}\right|$ is assumed to be approximately the width at half maximum of the wave function squared at one moment time t, one finds $\left|\Delta^{\prime} \mathrm{q}\right| \cdot\left|\Delta^{\prime} \mathrm{k}\right| \approx 1$ [Merzbacher, 9]. The discussion for time dependent $\mathrm{H}$ follows below, part I to part VI. 
I. One finds $\Delta^{\prime}$ variances can be regarded having equal values as $\Delta$ variations in time for $\mathrm{H}$ time dependent events and $\left|\Delta^{\prime}\right|$ equals $|\Delta|$, following [ref. 3]. Since the above is valid for any $\mathrm{t}$ in the domain $\Delta \mathrm{t}$, while keeping this $\mathrm{t}$ unchanged, it is now inferred that a relation $\left|\Delta \mathrm{k}_{-}\right| \cdot|\Delta \mathrm{q}| \approx \mathrm{c}_{-}$is valid during $\Delta \mathrm{t}$ for $\mathrm{H}$ time dependent events within the time interval description. It is derived in part $\mathrm{V}$ that the variable $\mathrm{c}_{-}$equals $\mathrm{h}+\mathrm{h}$. The writing order of the two variations in this relation is reversed compared to the above time moment description to remain consistent with and following the arguments in [ref. 3]. The $\Delta$ variations in time for $\mathrm{k}$ and $\mathrm{k}_{-}$are assumed to be the same. In that case $\Delta \mathrm{q}$ relates to $\Delta \mathrm{t}$ through $\mathrm{MV}$ comparative equilibrium and $\Delta \mathrm{k}_{-}$and $\Delta \mathrm{k}$ relate to $\Delta \mathrm{q}$ through relation $\left|\Delta \mathrm{k}_{-}\right| \cdot|\Delta \mathrm{q}| \approx \mathrm{c}_{-}$. When $\mathrm{H}$ time independent $\mathrm{c}_{-}=$ 1.

II. Time interval $\Delta \mathrm{t}$ is asymmetric when $\mathrm{H}$ time dependent, [ref. 3], and the domains $\Delta \mathrm{q}$ and $\Delta \mathrm{k}$ follow $\Delta \mathrm{t}$. The time dependence of the weight-function $\mathrm{w}(\mathrm{k})$ depends on the change of the free wave components during $\Delta \mathrm{t}$.

When one assumes $\mathrm{w}(\mathrm{k})(\mathrm{t})$ can be approximated without asymmetry and with a zero slope Heaviside block invariant in time, then $\mathrm{k}_{\text {_ }}$ equals center a, similar with time independent situations. It follows $\left|\Delta \mathrm{k} \_\right|$. $|\Delta \mathrm{q}| \approx \mathrm{c}_{-}$with $\mathrm{c}_{-}=1$ and trivially $\mathrm{w}(\mathrm{k})(\mathrm{t})=\mathrm{w}(\mathrm{k}(\mathrm{t}))=\mathrm{w}(\mathrm{k})$.

III. When one assumes $\mathrm{w}(\mathrm{k})(\mathrm{t})$ is asymmetric during the event time interval $\Delta \mathrm{t}$ it follows any time development of $\mathrm{k}(\mathrm{t})$ and $\mathrm{w}(\mathrm{k})(\mathrm{t})$ results in asymmetry for $\mathrm{w}(\mathrm{k})$ when $\mathrm{w}(\mathrm{k})$ can be approximated with a slope Heaviside block function:

Assume a linear approximation for $\mathrm{w}(\mathrm{k})$ : $\mathrm{w}(\mathrm{k})=(\mathrm{w} 0+\mathrm{w} \cdot \mathrm{k})$. Heaviside $(|\mathrm{k}-\mathrm{a}|-\mathrm{u})$ while $\mathrm{w}(\mathrm{k})$, including an invariant $\mathrm{w} 0$ and a slope $\mathrm{w}$ ' independent of $\mathrm{k}$, remains the same function for the complete $\mathrm{k}$-domain and for time interval $\Delta \mathrm{t}$ with time dependence through $\mathrm{w}(\mathrm{k})=\mathrm{w}(\mathrm{k}(\mathrm{t}))$. Necessarily center a remains invariant during $\Delta \mathrm{t}$ since $\mathrm{w}(\mathrm{k})$ is normalized and similarly $\mathrm{k}$ _ remains invariant however differs from a: for instance for $w^{\prime}>0$ there is $k_{-}{ }^{\wedge} 2=2 a^{\wedge} 2$ independent of $u$ and $w^{\prime}$. Within this approximation a change of $\mathrm{w}^{\prime}$ by multiplication with a positive real number is allowed however this is not considered due to center a remaining unchanged. Sign change for $\mathrm{w}^{\prime}$ is considered in part IV.

The domains $\Delta \mathrm{k}$ and $\Delta \mathrm{t}$ are related through $\left|\Delta \mathrm{k}_{-}\right| .|\Delta \mathrm{q}| \approx \mathrm{c}_{-}$during MV equilibrium where variation $\left|\Delta \mathrm{k}_{-}\right| \approx$ variation $|\Delta \mathrm{k}|$ following the discussion from part I. Slope w' can be introduced and interpreted, from this relation, to be the $\Delta \mathrm{k}$ comparative derivative of $\mathrm{w}(\mathrm{k})$ when the time interval asymmetry is added to the $\mathrm{k}$ domain asymmetry. This is possible for any partial $\Delta \mathrm{k}$ comparative derivative or partial one value $\mathrm{k}$ derivative of a linear function. All time interval dependent asymmetry for $\mathrm{k}$ included in the Heaviside part of $\mathrm{w}(\mathrm{k})$ remains zero because the Heaviside function itself remains invariant in time and a and $\mathrm{u}$ remain invariant in time. In this approximation $\mathrm{w}(\mathrm{k})(\mathrm{t}) \approx \mathrm{w}(\mathrm{k}(\mathrm{t}))$ for any $\mathrm{t}$ during $\Delta \mathrm{t}$.

IV. When one assumes $\mathrm{w}(\mathrm{k})$ changes during $\Delta \mathrm{t}$, ie one assumes $\mathrm{w}(\mathrm{k})=\mathrm{w}(\mathrm{k})(\mathrm{t})$ does not remain the same function, and the sign of the slope $\mathrm{w}^{\prime}=\mathrm{w}^{\prime}(\mathrm{t})$ changes during $\Delta \mathrm{t}$. This implies center a changes within the $\mathrm{k}$-domain and average momentum $\mathrm{k}$ _ changes accordingly during $\Delta \mathrm{t}$. A change of w' can only be assured by measurement. For instance a change from a to the opposite $-\mathrm{a}$, when reflection occurs of the original wave during $\Delta \mathrm{t}$ and momenta $\mathrm{k}$ and $\mathrm{k}_{-}$change to their opposites.

It depends on the sign of w' whether $\left|\Delta \mathrm{k}_{\_}\right| \cdot|\Delta \mathrm{q}| \approx \mathrm{c}_{-}$is more or less than 1 . When a sign change occurs during $\Delta \mathrm{t}$ and one assumes the $\Delta \mathrm{t}$ time interval average $\left\langle\mathrm{c}_{-}\right\rangle=1$, the description is within average similar to the time independent situation with w' equal to zero. 
Now approximated is $\mathrm{w}(\mathrm{k})$ with average $<\mathrm{w}$ ' $>$ equal to zero, even though for spin like properties this is not valid. In the time interval description the average $\left\langle w^{\prime}>=w^{\prime}\right.$ since the asymmetry is only in the non Heaviside part of $\mathrm{w}(\mathrm{k})$ and it follows w' equals zero and is time independent. In this approximation again trivially $\mathrm{w}(\mathrm{k})(\mathrm{t}) \approx \mathrm{w}(\mathrm{k}(\mathrm{t}))$. For spin like properties, instead of averaging $\mathrm{c}_{-}$, one can leave the time moment description unapplied completely and maintain a time interval only description, where a change of w' during $\Delta \mathrm{t}$ needs further interpretation.

V. When the relation $E=h . \omega$ can be justified for $w(k)$ for all situations, except yet for those with spin like properties, this can be regarded as an argument for the validity of relation $\mathrm{E}=\mathrm{h} . \omega$ for all non zero mass situations, $\mathrm{H}$ time dependent or $\mathrm{H}$ time independent in both the time moment description and in the time interval description, since one relation is expected to describe all non zero mass situations, just like one relation $\mathrm{E}=$ h. $v$ describes all zero mass situations, to remain with the concept of complementarity. For a time dependent situation particle momentum is not constant, and not necessarily $|\Delta \mathrm{E}|<<|\mathrm{E}|$ and one can approximate $\left|\Delta \mathrm{k}_{-}\right| \approx\left|\mathrm{k}_{-}\right|$and $|\Delta \mathrm{E}| \approx|\mathrm{E}|$. When considering a variable unspecified $\mathrm{h}$ ' instead of $\mathrm{h}$, there is $\mathrm{E}=\mathrm{h}^{\prime} . \omega$ and $\mathrm{p}=\mathrm{h}^{\prime} . \mathrm{k}_{-}$, and one has to apply a variable $\mathrm{c}_{-} \neq 1$.

From $\left|\Delta \mathrm{k}_{-}\right| \cdot|\Delta \mathrm{q}| \approx \mathrm{c}_{-}$and with a variable $|\Delta \mathrm{p}| \cdot|\Delta \mathrm{q}|=\mathrm{h}+$, like in paragraph 2 , there is $|\Delta \mathrm{p}| \approx \mathrm{h}+/ \mathrm{c}_{-} \cdot\left|\Delta \mathrm{k}_{-}\right|$ and for $\Delta \mathrm{p} \approx-\mathrm{p}$ and $\Delta \mathrm{q} \approx-\mathrm{q}$ there is $|\mathrm{p}| \approx \mathrm{h}+\mathrm{c}_{-}\left|\mathrm{k}_{-}\right|$. The estimates for variations $\Delta \mathrm{p}$ and $\Delta \mathrm{q}$ are derived in [ref. 3]. When one assumes $c_{-}=\mathrm{h}+\mathrm{h}>0$, a variable, to maintain the complementarity relation $\mathrm{p}=\mathrm{h}$. $\mathrm{k}_{-}$, one can insert h' $=\mathrm{h}$ and it follows $\mathrm{E}=\mathrm{h} . \omega$, and $\mathrm{E}>=0$ as it should be for a "kinetic" energy, and $\left|\Delta \mathrm{k}_{-}\right| \cdot|\Delta \mathrm{q}| \approx \mathrm{h}+\mathrm{h}$ which is variable for $\mathrm{H}$ time dependent however equal to one, $\mathrm{c}_{-}=1$, for $\mathrm{H}$ time independent.

VI. The exceptional occurrence of $\mathrm{c}_{-}=0$ and $\mathrm{h}+=0$, means $\Delta \mathrm{E}=-\mathrm{h}+/ \Delta \mathrm{t}=0$, ie the kinetic energy $\mathrm{E}=\mid \mathrm{h}$. 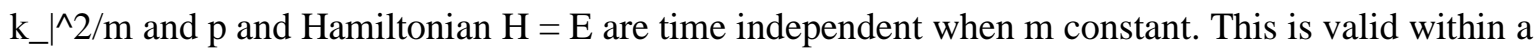 Lagrangian equilibrium description for the associated wave particle with a space coordinate equal to $\mathrm{q}=$ (h. $\mathrm{k} \_/ \mathrm{m}$ ). $\mathrm{t}$ and constant velocity $\mathrm{dq} / \mathrm{dt}$, see above. In this situation from $\mathrm{h}+=0$ one finds the commutator $[\mathrm{p}, \mathrm{q}]=2 \mathrm{p}$. q. One can derive the corresponding time interval description commutator, later defined in paragraph 5, equals $|E / \Delta E| h+$. In paragraph 5. it is argued that $h$ is a limit for $h+$ when $H$ time independent, and it follows $\mathrm{c}_{-}=0$ would mean $\mathrm{h}$ equals zero and the limit of $\mathrm{qm}$ is reached, still assuming $\mathrm{m}$ is an invariant. In paragraph 6 . it is argued that the fundamental qm commutator relation for operators for $\mathrm{p}$ and $\mathrm{q}$ remaining constant within the time moment description with Lagrangian equilibrium, has to be re-evaluated within the time interval description with MV equilibrium. However c_being equal to zero is not further studied in this paper.

The time interval description including comparative derivatives depends, due to the MV theorem, on coordinate differences and asymmetry during $\Delta \mathrm{t}$. Measuring properties during $\Delta \mathrm{t}$ when $\mathrm{H}$ is time dependent is possible and $\Delta \mathrm{t}$ can include measurements, of which the time order can be defined however their time dependence only by the "during $\Delta t$ " indication when remaining within a time interval only description. The relation of coordinates and intervals remains a subject of study. [ref. 3] includes a discussion of the interpretation of time interval boundaries and the definition of time intervals with respect to the asymmetric time coordinate definition that includes the connectedness property and time order.

\section{Field properties}


Of the field theory concepts newly defined for the time interval version, operators are the main focus. The following concepts and definitions within the time moment description are mostly from [Roos, 4] and [Merzbacher, 9]. They remain of value for the time interval description.

\section{Observables}

From Newtonian mechanics the definition of "free" for a non zero mass particle means without interference and includes momentum p conservation. Within qm, no interference means the wave function is a linear combination of "free" or "plane" waves and wave momentum $\mathrm{k}$ is conserved for each of the free waves. Average wave momentum $\mathrm{k}_{-}$and complementary particle momentum $\mathrm{p}$ are related through $\mathrm{h}$, the constant of Planck.

"free" solutions of the field, or wave, equation assuming Lagrangian equilibrium remain "free" when they are "dispersion" free. Dispersion free is similar to conservation of observable value: the solution is, by the corresponding operator, transformed to itself.

\section{Field limit process}

Within field theory specific paths with time moment depending coordinates are disregarded [Beller, 10], rather it relates to a "field" throughout space including waves that remain complementary to an equivalent wave particle description. Fields and some field properties are non localizable, and in this way for instance allow that the wave function can collapse to a, more local, place in space time.

Fields are usually introduced with the help of the following construction [De Witt, Smith, 11], [Sakurai, 12]: one defines a for instance linear space filled with all similar parts, each connected with the next one, where at each space coordinate the field energy is a functional of the part properties and their connections. The choice for the field energy related to radiation waves is discussed for instance in [Sakurai, 12], [Jauch, Rohrlich, 13], and below in paragraph 9.

This is only one of many possible constructions and its choice depends on whether a description of reality is found consistent with measurements. Basic is that "every" part of space for all space coordinate values is also part of the field, when the field limit process, the limit process of increasing number of parts and decreasing distances, is considered, thus creating the field.

The concept of a field "through all space" and including energy at every place in space is questionable. One of its motivations is the possibility to construct the usual creation and destruction operators where the field energy can be expressed with integers.

\section{Operators}

Operators work on observables by a particular activity. With a time interval one can describe change and differences during a time interval event, with the usual one moment time one regards change from the perspective of one moment. Operators can be defined by their commutator properties and some operators with the help of derivatives [Sakurai, 12], [Jauch, Rohrlich, 13]. Specific time interval related comparative commutators are defined in paragraph 5, to introduce the time interval description of operators.

Operators for existing field theory, within the time moment description, are indicated with and work on expressions, within square brackets to the right of them, which can include other operators. Commutators are written with square brackets with two entities divided by a ",". Like for all constants, $\mathrm{h}$ implies multiplication with $\mathrm{h}$, the constant of Planck. The fundamental equation $\left[\mathrm{p}^{\sim}(\mathrm{t}), \mathrm{q}^{\sim}(\mathrm{t})\right]=-$ ih depends on $\left[\mathrm{h}^{\sim}\right.$, $\left.\mathrm{q}(\mathrm{t})^{\sim}\right]=0$, which is assured by h having an invariant value. Definitions equivalent to the commutator version include: $\mathrm{q}^{\sim}$ [function] $=\mathrm{q}$. function, and $\mathrm{p}^{\sim}[$ function] $=-\mathrm{ih} \partial / \partial \mathrm{q}($ function $)$, where $\partial / \partial \mathrm{q}$ is the 
partial derivative to $\mathrm{q}$ for $\mathrm{q}=\mathrm{q}(\mathrm{t})$ at a certain one moment time $\mathrm{t}$. The factor $-\mathrm{ih}$ depends on the normalization of the wave function from complementarity.

\section{Operators and the time interval description}

Within the time interval description of field theory, operators are defined now by applying "time interval" or "comparative" commutators and time interval derivatives. Especially when $\mathrm{H}$ is time dependent, for instance when wave function collapse occurs during a time interval event, the time interval description differs from the time moment description.

The event of star source radiation emission, and successive wave function collapse as part of a measurement, while $\mathrm{H}$ is time dependent, is discussed by application of the time interval description in [ref. 3]. Several relations for $\mathrm{H}, \mathrm{p}, \mathrm{q}$ and $\Delta \mathrm{t}$ are derived there that are now assumed to be valid also for other $\mathrm{H}$ time dependent situations. This consideration depends on star source radiation waves being basic and exemplary: the wave function being a linear combination of free waves, and a zero mass complementary particle. Since relations $\mathrm{E}=\mathrm{h} . \mathrm{v}$ for zero mass and $\mathrm{E}=\mathrm{h} . \mathrm{\omega}$ for non zero mass have the same expression, paragraph 2 , it is assumed, within the time interval description and when $\mathrm{H}$ time dependent, some relevant relations for zero mass radiation waves can be generalized to non zero mass matter waves.

Time coordinates t do not always commute, except the specific time interval boundary coordinates ta and $\mathrm{tb}=\mathrm{tb}(\mathrm{ta})$. The definition of "comparative" commutators for the time interval $\Delta \mathrm{t}=[\mathrm{tb}, \mathrm{ta}]$ and any quantities $\mathrm{A}$ and $\mathrm{A}$ ' applies vertical bars to indicate the time interval:

1. $\quad\left[\mathrm{A}^{\prime}, \mathrm{A}\right] \| \Delta \mathrm{t}=1 / 2\left[\mathrm{~A}^{\prime}(\mathrm{tb}) \cdot \mathrm{A}(\mathrm{tb})-\mathrm{A}(\mathrm{ta}) \cdot \mathrm{A}^{\prime}(\mathrm{ta})\right]$

When "mean velocity theorem" $\mathrm{MV}$ equilibrium is assumed during $\Delta \mathrm{t}$, and A' $=1 / \mathrm{t}$ it follows: [A', $\mathrm{A}] \| \Delta \mathrm{t}$ $=$ "dA $/ \mathrm{dt}$ " $\mid \Delta \mathrm{t}$, ie equal to the $\Delta \mathrm{t}$ time interval derivative of A. Any derivative to time interval $\Delta \mathrm{t}$, while assuming MV equilibrium, can be written applying comparative commutators, [ref. 3]:

2. “dA/dt” $\left\|\Delta \mathrm{t}=\left[\mathrm{A}^{\prime}, \mathrm{A}(\mathrm{t})\right]\right\| \Delta \mathrm{t}=1 / 2(1 / \mathrm{tb} . \mathrm{A}(\mathrm{tb})-\mathrm{A}(\mathrm{ta}) .1 / \mathrm{ta})$

Comparative operators are indicated with * and work on the expression between brackets on the right of them. For all parameters like space coordinates q: operator $\mathrm{q}^{*}$ means multiplication with $\mathrm{q}$, similar like within the one moment time description. Other operators like $\mathrm{E}^{*}$ and $\mathrm{p}^{*}$ for energy and momentum need definition each from equation 3. newly. The operator definition follows from the generalization of time interval relations for radiation wave events. It emphasizes the "change during time interval event" character of operators, applying time interval derivatives " $\mathrm{d} / \mathrm{dt}$ " $\mid \Delta \mathrm{t}$ or alternatively comparative commutators. With equation 3. "comparative" operators within the time interval description are defined, depending on time interval $\Delta \mathrm{t}$ however without one moment time parameter $\mathrm{t}$ and without the constant of Planck h. They work on any entity A, writing A with brackets: [A]. The meaning of "." is ordinary numerical multiplication.

3. $\quad$ operator* $[\mathrm{A}]=$ "dd/dt”(|operator*|. A) ||$\Delta \mathrm{t} . \Delta \mathrm{t}$ 


$$
=\left[\mathrm{A}^{\prime}, \mid \text { operator* } * \text {. A }\right]|| \Delta \mathrm{t} . \Delta \mathrm{t}
$$

$\mid$ operator* $\mid=$ corresponding quantity for the operator. E.g., |operator*| equals quantity $\mathrm{p}$ when operator* equals operator $\mathrm{p}^{*}$, ie $\left|\mathrm{p}^{*}\right|=\mathrm{p}$. The first definition applies a time interval derivative “ $\mathrm{d} / \mathrm{dt}$ ” ||$\Delta \mathrm{t}$ working on |operator*|. A, the second one a comparative commutator. A' in equation 3. equals the $1 /$ t quantity, however the comparative commutator only applies tb and ta following equation 2. From equation 3. follow the relations:

4. $\mathrm{h}+*[\mathrm{~A}]=\left[\mathrm{A}^{\prime}, \mathrm{h}+\mathrm{A}\right] \| \Delta \mathrm{t} . \Delta \mathrm{t}$

$\mathrm{p}^{*}[\mathrm{~A}]=\left[\mathrm{A}^{\prime}, \mathrm{p} . \mathrm{A}\right] \| \Delta \mathrm{t} . \Delta \mathrm{t}$

$\mathrm{E}^{*}[\mathrm{~A}]=\left[\mathrm{A}^{\prime}, \mathrm{E} . \mathrm{A}\right] \| \Delta \mathrm{t} . \Delta \mathrm{t}$

The operators $\mathrm{p}^{*}$ and $\mathrm{E}^{*}$ reduce to $\mathrm{p}^{\sim}$ and $\mathrm{E}^{\sim}$ when $\mathrm{H}$ is time independent and indeed in that case one finds commutator definitions, and derivative definitions, to be the same for the time interval description and for the time moment description. In that case $\mathrm{p}^{*}$ equals $\mathrm{p}^{\sim}$ and $-\mathrm{ih} \partial / \partial \mathrm{q}$ and $\mathrm{E}^{*}$ equals $\mathrm{E}^{\sim}$ and $+\mathrm{ih} \partial / \partial \mathrm{t}$. Time interval operators differ from time moment operators when $\mathrm{H}$ is time dependent.

The constant of Planck is a limit for $\mathrm{h}+$ when $\mathrm{H}$ is time independent and this provides some meaning to it. $\mathrm{h}+$ and $\mathrm{h}$ - can be interpreted to represent the energy asymmetry during $\Delta \mathrm{t}$, and the relation $\mathrm{E}=\mathrm{h}+/ \Delta \mathrm{t}$, a relation derived for radiation waves, can be generalized to be valid for all $\mathrm{H}$ time dependent situations. MV comparative equilibrium depends on variation during the complete time interval $\Delta t$ rather than on the derivation limit at a specific one moment time and it can evaluate asymmetry of properties during $\Delta \mathrm{t}$. $\mathrm{h}+\mathrm{is}$ derived from MV comparative equilibrium for $\Delta \mathrm{t}$ and is not a constant when $\mathrm{H}$ is time dependent, however for $\mathrm{H}$ time independent, without asymmetry during $\Delta \mathrm{t}, \mathrm{h}+$ reduces to $\mathrm{h}$.

\section{Space time equilibrium translations and Lorentz transformations, and metric tensor space time dependence}

The causality interpretation of comparative commutator relations for any comparative operators $\mathrm{A}^{\text {** and }}$ $A^{*}$ is expected to be the same as for commutator relations for operators $A^{\sim \sim}$ and $A^{\sim}$ and the same for $\mathrm{H}$ time independent and $\mathrm{H}$ time dependent events. Several arguments for this inference follow from the discussion of transformations within the time interval description including MV equilibrium given in [ref. $3]$.

From time interval operator equation 3 , when $\mathrm{H}$ is time dependent and $\mathrm{h}+\neq \mathrm{h}$, it follows:

5. $\left[\mathrm{E}^{*}, \mathrm{q}^{*}\right]|| \Delta \mathrm{t}\left[\right.$ function] $=-2 \mathrm{q}(\mathrm{t} 0) \mathrm{t} 0 \mathrm{p}(|\Delta \mathrm{q} / \Delta \mathrm{t}||\Delta \mathrm{t}|-|\partial \mathrm{q} / \partial \mathrm{t}|) .(1 / \mathrm{t})^{\wedge} 2 \Delta \mathrm{t}$. (function)

The time t0 is the time unit for multiplication, necessary to define time coordinates properly [ref. 3], and $\mathrm{q}(\mathrm{t} 0)$ is the corresponding space coordinate when maintaining MV equilibrium. $\mathrm{t} 0$ is not by definition the zero time coordinate in space time and $\mathrm{q}(\mathrm{t} 0)$ is not by definition equal to $\mathrm{q} 0$, the zero space coordinate in space time. $t 0$ is "neutral" since multiplication with t0 leaves the past or future property of any coordinate $\mathrm{t}$ unchanged. Assuming $|\mathrm{p}| \neq 0$, the comparative commutator for $\mathrm{E}^{*}$ and $\mathrm{q}^{*}$ is non zero. 
For events with $\mathrm{H}$ time dependent $\mathrm{E}$ equals $\mathrm{h}+/ \Delta \mathrm{t}$ and an equation with $\mathrm{h}+*$ is valid similar to equation 5 . with $\mathrm{E}^{*}$ and it follows $\left[\mathrm{h}+^{*}, \mathrm{q}^{*}\right]$ is non zero implying $\left[\mathrm{p}^{*}, \mathrm{q}^{*}\right] \| \Delta \mathrm{t}$ differs from $\left[\mathrm{p}^{\sim}, \mathrm{q}^{\sim}\right]$. Comparative commutator $\left[\mathrm{p}^{*}, \mathrm{q}^{*}\right] \| \Delta \mathrm{t}$ is part of the construction of the time interval description operators $\mathrm{a}^{*}, \mathrm{a}+^{*}$ and $\mathrm{Nk}^{*}$ equivalent to the usual time moment description operators. Even so, number operator $\mathrm{Nk}^{*}$ can be normalized and its values can still be integers.

When $\mathrm{H}$ time independent space translation invariance is consistent with the definition of [tb, ta] where tb depends on $\mathrm{ta}$, with $\mathrm{tb}=\mathrm{tb}(\mathrm{ta})$, while assuming MV equilibrium [ref. 3]. When $\mathrm{H}$ time dependent, from equation 5. and from the triangle inequality [Hocking, Young, 1], one derives that an equilibrium translation $(\mathrm{q}, \mathrm{t})$ to $(\mathrm{q} ", \mathrm{t}$ ") $=(\mathrm{q}+\mathrm{qL}, \mathrm{t}+\mathrm{tL}))$ implies derivative difference

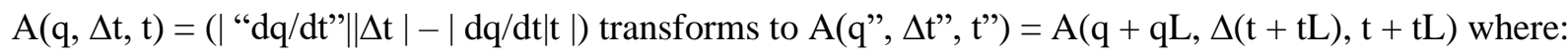

6.

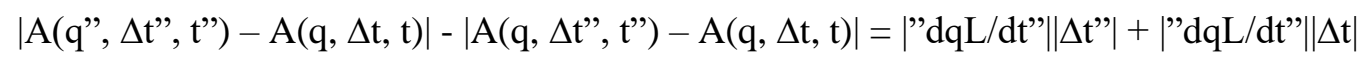

This is a situation where the triangle inequality allows an equal sign. The ordinary one moment time $t$ derivative for $\mathrm{q}$ is written with $\mathrm{dq} / \mathrm{dt} \mid \mathrm{t}$, with one bar. From equation 6 . it follows that $\mathrm{A}(\mathrm{q}$ ", $\Delta \mathrm{t}$ ", $\mathrm{t}$ ") is linear in $\mathrm{qL}$, or equivalently in $\mathrm{tL}$, for infinitesimal equilibrium translations, with $\mathrm{tL} / \mathrm{t}<<1$ and $\mathrm{qL} / \mathrm{q}<<1$, and quadratic like $\mathrm{qL} . \mathrm{tL}$ otherwise, with $\mathrm{tL} / \mathrm{t} \gg 1$ and $\mathrm{qL} / \mathrm{q} \gg 1$. Both the right side time interval derivative terms in equation 6 . are non zero and they are not the same, because time interval $\Delta t=[\mathrm{tb}$, ta] is not time translation invariant and is asymmetric when $\mathrm{H}$ is time dependent. They are specific for the time interval description, one moment time derivatives similar to these are equal to zero. The meaning of a constant when applying derivatives changes within the time interval description. Translation invariance for space time is not always valid when $\mathrm{H}$ time dependent.

Except for situations with zero mass and the light velocity property, writing c_light for the usual $c$, where the metric space time path length is zero, metric space time distance is not always conserved by an equilibrium translation or by the above transformation. Lorenz transformations however by definition keep metric distances unchanged. When a transformation of $\mathrm{A}$, linear in $\mathrm{qL}$ or $\mathrm{tL}$, from equilibrium translation (q, t) to (q", t") can be interpreted as an approximation to a infinitesimal Lorentz transformation, this indicates that, or: the usual time moment description in space time coordinates of Lorentz transformations should be reviewed, or: to keep metric distances unchanged the metric tensor is to be space time dependent, a property that does not itself depend on the description, with time intervals or time moments. With the introduction of time intervals [tb, ta] for $\mathrm{H}$ time dependent in [ref. 3], both time and space coordinate translation invariance in equilibrium can become lost.

\section{Discussion: Radiation waves and matter waves, and gravitation for zero and non zero mass}

In the following considered are events where the mass $m=m(t)$ varies depending on $t$, including partially through $\mathrm{q}$ when $\mathrm{q}=\mathrm{q}(\mathrm{t})$ : the domain for time, interval $\Delta \mathrm{t}=\Delta 1 \mathrm{t}=[\mathrm{tb}, \mathrm{ta}]$, is equivalent to the domain for space, interval $\Delta \mathrm{q}=\Delta 1 \mathrm{q}$ in the time interval description. For radiation waves emitted by a star source two successive time interval events $\Delta 1 \mathrm{t}$ and $\Delta 2 \mathrm{t}$ can be defined. The subscript 1 is applied for $(\Delta 1)$ domains for the time interval $\Delta 1 \mathrm{t}$ event of wave propagation from the star source at qb to the wave propagation surface that includes a space coordinate $q a$. The space parameter $q=q(t)$ is chosen such that $|q|$ is a surface radius measure and with $q$ meant is in fact the space interval $\Delta 1 \mathrm{q}$ that can be chosen $[\mathrm{qb}, \mathrm{q}]=[\mathrm{q}(\mathrm{tb})$, $\mathrm{q}]$ for any $\mathrm{q}$ on the propagation surface. In this way one remains within the time interval description, including related 
interval coordinate domains. One can write $\Delta 1 \mathrm{q}=[\mathrm{qb}, \mathrm{qa}]$ by choosing $\mathrm{q}(\mathrm{ta})=\mathrm{qa}$ along a specific "line of sight" radius for all different $\Delta 1 \mathrm{t}$.

In the next paragraph the subscript 2 is applied for $(\Delta 2)$ domains for the time interval $\Delta 2 \mathrm{t}$ event of wave function collapse from the during $\Delta 1 \mathrm{t}$ reached propagation surface to a measurement place at the specific site qa at this propagation surface. Whenever $\Delta 1 \mathrm{t}$ changes in duration with radius $|\mathrm{q}|$ the related propagation surface and $\Delta 1 \mathrm{q}$ change accordingly, including site qa. Since it is assumed $|\mathrm{qa}-\mathrm{q} 0|<<|q b|$ this means space origin q0 is chosen to change with qa and $\Delta 1 \mathrm{t}$. From the chosen place in space for q0 follow the values of other space coordinates including those for qb.

Radiation waves imply zero mass wave particles, however for waves with non zero mass wave particles, properties are generalized from radiation wave properties, like discussed in paragraph 5. From the complementarity relations, paragraph 2 , applying comparative derivatives, and with $\mathrm{k} \_$the average wave momentum, one finds:

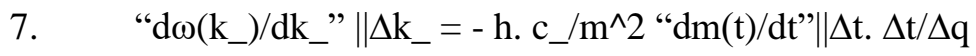

There is $\left|\Delta \mathrm{k}_{-}\right| \cdot|\Delta \mathrm{q}| \approx \mathrm{c}_{-}$for $\Delta$ variations in time just like the usual $\mathrm{H}$ time independent equation $\left|\Delta^{\prime} 1 \mathrm{k}\right|$. $\left|\Delta^{\prime} 1 \mathrm{q}\right| \approx 1$, paragraph 3. Variation $\Delta \mathrm{k}_{-}$means variation of average momentum $\mathrm{k}_{-}$, during time interval $\Delta \mathrm{t}$, that includes variation of all momenta $\mathrm{k}$ of its wave components. The generalization from radiation wave properties to matter wave properties is now applied to infer equation 7. is valid for all non zero mass wave particles for events with $\mathrm{H}$ time dependent.

For $\mathrm{H}$ time independent and $\mathrm{h}+\mathrm{h}$, for a stationary state wave propagation confirmation measurement, the uncertainty relation $|\Delta \mathrm{E}| .|\Delta \mathrm{t}| \approx \mathrm{h}$ or $|\Delta \mathrm{E}| \approx \mathrm{h} /|\Delta \mathrm{t}|$ assures the measurement uncertainty for $|\mathrm{E}|$ is much less than the value for $|E|$ itself, while providing a measurement requirement for duration $|\Delta t|$, [Merzbacher, 9]. However wave propagation time interval $\Delta 1 \mathrm{t}$ does not include measurements, wave function collapse and measurement occur during $\Delta 2 \mathrm{t}$. For time interval $\Delta 1 \mathrm{t}, \mathrm{H}$ remains time independent although the stationary state measurement requirement does not remain valid and there is no need for the time interval duration $|\Delta 1 \mathrm{t}|$ to be chosen such that variation $|\Delta 1 \mathrm{E}|$ is consistent with the above "stationary state" property since $|\Delta 1 \mathrm{E}|$ is a variation during time interval $\Delta 1 \mathrm{t}$, not a measurement uncertainty. The necessary assumption $|\Delta 1 \mathrm{E}|=|\mathrm{E}|$ as a wave property can be saved and equation 7 . can be saved for $\Delta 1 \mathrm{t}$ even while $\mathrm{H}$ is time independent for this time interval.

Waves emitted by a star source are not consistent with plane waves. The stationary state property seems well justified for one-dimensional plane wave combinations invariant towards infinity. Star source wave propagation is described with propagation sphere surfaces and without preferred specific space coordinates on these surfaces. Both place and energy are non localizable during $\Delta 1 \mathrm{t}$, allowing the above wave energy variation property, equation 7 . Only during $\Delta 2 \mathrm{t}$ and because of wave function collapse localizable properties emerge. This indicates that $\Delta 1 \mathrm{t}$ has some properties similar to $\mathrm{H}$ time dependent and non stationary events, however $\Delta 1 \mathrm{t}$ remains a $\mathrm{H}$ time independent time interval, during which no external interference occurs. During time interval $\Delta 2 \mathrm{t}, \mathrm{H}$ is time dependent including external interference, wave function collapse and a measurement place with specific space coordinates a-symmetric relative to the star source symmetry. 
Considering this situation for $\Delta 1 \mathrm{t}$ and applying to $\Delta 1 \mathrm{t}$ properties for $\mathrm{H}$ time dependent radiation wave events, generalized to include non zero mass complementary particle events, one can apply $c_{-}=h+/ h$ from paragraph 3. Still $\mathrm{H}$ remains time independent with $\mathrm{h}+=\mathrm{h}$ and $\mathrm{c}_{-}=1$ for $\Delta 1 \mathrm{t}$, and for non zero mass $\mathrm{m}$ it follows:

8. “ “dE/dt”|| $1 \mathrm{t}=+(\Delta 1 \mathrm{q} / \Delta 1 \mathrm{t})^{\wedge} 2 . " \mathrm{dm} / \mathrm{dt} "|| \Delta 1 \mathrm{t}$

$$
\text { “dE/dt” }\left|\Delta 1 \mathrm{t}=+(\Delta 1 \mathrm{q} / \Delta 1 \mathrm{t})^{\wedge} 3 \cdot “ \mathrm{dm} / \mathrm{dq} "\right| \mid \Delta 1 \mathrm{q}
$$

Energy $E=$ h. $\omega$ is a non zero mass particle associated wave energy and the propagation surface wave energy is $E s=n \# . h . \omega$, where $n \#$ is the number of complementary particles. Equation 8, a result of equation 7, could also be derived as a consequence of the MV equilibrium theorem that assures the time interval $\Delta 1 \mathrm{t}$ derivative of $\mathrm{q}$ in MV equilibrium is a constant.

In [ref. 3] it is described that in contrast to one moment time derivatives a time interval derivative of a constant function not necessarily is zero. This is of relevance for the interpretation of equation 8 .

\section{Wave energy Es remains invariant and mass $m$ remains zero: radiation waves}

During $\Delta 1 \mathrm{t}$, and when $\mathrm{m}$ remains zero, the situation resembles radiation waves without external interference and with an average wave momentum $\mathrm{k}$ _ remaining invariant. Valid is propagation surface wave energy Es $=\mathrm{n} \#$. h.v, $\mathrm{n} \#$ the wave particle number, while for $\mathrm{m}$ equals zero equation 8 . is not valid. Interpretation as a field energy depends on the radiation wave propagation surface energy Es being similar to the usual choice for the field energy within the time moment description, paragraph 4, [Sakurai, 12]. During $\Delta 2 t$ there is external interference like for instance the photo-electric effect interaction. Because of external interference, Es and wave particle number $n \#$ both are variable, however frequency $v$ remains invariant.

\section{Wave energy Es and field energy Ee: metric surface area requirement}

Consider again $\mathrm{m}$ constant and zero and $\Delta 1 \mathrm{t}$ the relevant time interval. With $\mathrm{H}$ time independent, $\Delta 1 \mathrm{t}$ remains without external interference and can be interpreted with: zero mass radiation wave propagation with the c_light property $(\Delta \tau)^{\wedge} 2=0$.

Propagation surface wave energy Es is assumed equal to surface field energy Ee $=\mathrm{E} 1$. A . $|\mathrm{d}| /|\mathrm{q}|$ with A the metric surface area and Ee constructed from the specific surface curvature energy defined in [ref. 6], where it is further assumed $|\mathrm{d}|=|\Delta \mathrm{lq}|=|\mathrm{q}|$ and thus Ee $\sim$ A. Below and in paragraph 8. the assumption Es equals, can be interpreted by, the field energy Ee is further justified. The propagation surface wave energies $\mathrm{E}$ and $\mathrm{Es}=\mathrm{n \#}$. E both should remain constant for all $|\mathrm{q}|$ for radiation waves emitted by a star source because of conservation of energy. This means A has to fulfill a metric surface area requirement, that is, to remain constant.

The field limit process, described in paragraph 4 , applies to any time or space interval measures $|\Delta 1 \mathrm{t}|$ or $|\Delta 1 \mathrm{q}|=|\mathrm{q}|$. The validity of the metric surface area requirement is equivalent to the valid and consistent application of the field limit process to construct the field energy Ee. Due to this, field energy Ee, which is proportional to the metric 2-surface area A, remains constant for all $\Delta 1 \mathrm{t}$ and this means validness of Es Ee, and equivalence of propagation surface wave energy invariance with metric propagation surface area invariance for all $\Delta 1 t$, when $\Delta 1$ t and propagation surface radius $|q|$ vary within comparative equilibrium. 
When the metric 2-surface area $\mathrm{A}$ is invariant and independent of $|\mathrm{q}|$ or $\Delta 1 \mathrm{t}$, the metric tensor mij is variable and dependent on $|\mathrm{q}|$ and $\Delta 1 \mathrm{t}$, ie on space time. This discussion differs from the result in paragraph 6, where infinitesimal derivative difference transformations and traditional Lorentz transformations are derived to be similar, including however like above a metric tensor mij expected to be variable with space time. Solutions for such a surface and metric requirement exist, for instance "wrinkled" surfaces. For any wrinkled or other solution it is pertinent that there are no double radii: for any q' at the propagation surface for time interval $\Delta 1 \mathrm{t},|\mathrm{q}|=\mid \mathrm{q}$ ' $-\mathrm{qb} \mid$ is the only radial distance from the surface to the source at qb along radius q' - qb and there is no other q" at the surface along this radius.

\section{Wave energy $E$ and mass $m$ variable: internal energy transfer and non localizable wave particle mass gravitation}

When $\mathrm{E}$ and a non zero $\mathrm{m}$ are both variable, the complementary wave momentum $\mathrm{k}_{\text {_ }}$ is variable and the wave components are not dispersion free. Internal energy transfer includes non Newtonian gravitational interaction with a change of mass $\mathrm{m}$. Mass $\mathrm{m}$ and wave number $\mathrm{n \#}$ exchange value when the propagation surface changes with $\Delta 1 \mathrm{t}$. Both wave energy $E$ and complementary particle mass $m$ change linear with $1 / q \mid$ when $|q|$ itself increases with $|\Delta 1 t|$ while similarly $k_{-}$changes linear with $1 / q \mid$. The propagation surface energy Es $=\mathrm{n \#}$. E remains necessarily constant so $\mathrm{n \#}$ should be proportional to $|\mathrm{q}|$. Recall q itself has the meaning of an interval in space [qb, qa], and $|\mathrm{q}|$ is the metric space distance for the specific surface "line of sight" radius. During $\Delta 1 \mathrm{t}$, when $\mathrm{E}$ and $\mathrm{m}$ variable, $|\mathrm{E}|$ and $\mathrm{m}$ both diminish due to propagation. "Far" away from the source, and only for a localizable (star) source, the non zero mass m matter waves emerge like, ie approach in properties, zero mass radiation waves, or, like an increasing number n\# of zero mass radiation wave particles, ie n\# photons.

Wave energy $E$ variable and mass $m$ invariant and non zero: localizable star source mass gravitation When $\mathrm{m}$ is non zero and invariant, this situation can be interpreted with Newtonian gravitation energy where a variable solution for surface field energy Ee exists, from equation 8, linearly proportional with $|q|$ $=\left|q^{\prime}-q b\right|=A \cdot\left(q b+q^{\prime}\right)^{\wedge}(-1)$ for any q' at the propagation surface. It can be argued, when the above metric propagation requirement for surface area $\mathrm{A}$ is valid for $\Delta 1 \mathrm{t}$, this means $\mathrm{Ee} \sim 1 / \mathrm{qb} \mid$. The metric requirement is a propagation surface area only requirement and valid also for non zero matter waves when constructing the field with energy Ee, and not to be applied to metric distances even when metric sphere surface area relates to metric radius distance squared traditionally with the traditional metric. Propagation sphere surfaces are due to waves emitted by a localizable (star) source. With other propagation concepts, for instance 1-space dimensional like a circular line, the field energy is a 1-space field energy and the normal dimension d changes accordingly and with it a metric requirement, if there is any. Stationary plane waves would require a flat propagation area. A realistic space time is assumed to necessarily be a $3+1$ space time, paragraph 8, comment III, and higher dimensional d normal to the field space can occur. Field energy Ee can be explained by assuming the existence of a non zero mass m' source centered at qb, which defines energy Ee as gravitational and defines $m$ as gravitational mass, both non localizable. $\Delta 1 \mathrm{t}$ includes a specific place at q' = qa, and with the choice for space origin q0 "near" qa: $|\mathrm{qa}-\mathrm{q} 0|=|\mathrm{qa}|<<$ $|q b|$, it follows Ee is linear in $1 /|q|$. The coordinates for $q b$ change accordingly with the propagation surface while the place of qb in space remains the same. Measurement place qa should change its place in space time together with the propagation surface and $\Delta 1 \mathrm{t}$ to remain a measurement place. Gravitational mass and wave kinetic inertial mass being the same $m$ is consistent with the complementary relation $\mathrm{p}=\mathrm{h}$. $\mathrm{k}_{-}$. Since during $\Delta 2 \mathrm{t}$ waves can interact with the measuring device at qa this can confirm whether energy emission 
at $\mathrm{qb}$ is due to a gravitational source $\mathrm{m}$ ' or to another source. The interpretation with gravitational mass depends on the source mass m' at qb. In paragraph 9, it is derived that for this field energy the Noether charge $\mathrm{Nc} \sim \mathrm{A}^{\wedge} 2$ and one can infer $\mathrm{A}$ to be proportional to the constant source mass, and the wave particle mass, $\mathrm{m}^{\prime} . \mathrm{m}$. This is consistent with the metric propagation surface area requirement for $\mathrm{A}$, and it is consistent with the surface curvature field energy Ee being proportional to $\mathrm{A} /|\mathrm{q}| \sim 1 / \mathrm{q} \mid$.

Following the discussion in paragraph 6. translation invariance for space is challenged during $\mathrm{H}$ time dependent time interval $\Delta 2 \mathrm{t}$ meaning the choice for origin $\mathrm{q} 0$ is not completely arbitrary. The relevant assumption for $\mathrm{q} 0$ is distance $|\mathrm{qa}-\mathrm{q} 0|<<|\mathrm{qb}|$. Lorentz transformations are defined to not change metric distances. When the Lorentz transformation definition remains valid and infinitesimal equilibrium derivative transformations are linear in equilibrium translations and can be regarded as infinitesimal Lorentz transformations according to paragraph 6 , the choice for origin $q 0$ with $|q a-q 0|<<|q|$ is a valid choice also for $\mathrm{H}$ time dependent time interval $\Delta 2 \mathrm{t}$. A change of the propagation surface and radius $|\mathrm{q}|$ together with $\Delta 1 \mathrm{t}$ and $\Delta 2 \mathrm{t}$ means the space origin choice $\mathrm{q} 0$ changes accordingly where relation $|\mathrm{qa}-\mathrm{q} 0|$ $\ll<\mathrm{q} \mid$ remains necessary. At least for infinitesimal equilibrium changes of $|\mathrm{q}|$ together with $\Delta 1 \mathrm{t}$, metric distances remain unchanged like for Lorentz transformations, and this relation remains valid.

\section{Summary: non zero and zero mass similarity}

The $1 /|q|$ inverse distance energy dependence is derived within the time interval description from the surface field energy Ee, for $\mathrm{m}$ non zero whether invariant or variable. For $\mathrm{m}$ non zero the above gravitation interpretations apply, from equations 8 . For $\mathrm{m}$ zero and invariant the surface field energy Ee is constant and the same as radiation wave propagation surface energy Es following the metric propagation surface area requirement. When the source emission value changes both Es and Ee change proportional to A when Noether charge $\mathrm{Nc}=\mathrm{A}^{\wedge} 2$ remains a valid estimate for how the inverse distance energy relation includes the relevant source property, meaning for matter waves: source mass $\mathrm{m}$ '. When wave momentum $\mathrm{k}_{-}$changes according to internal change of the source, both Es and Ee change proportional with A, through the property: wave particle mass $\mathrm{m}$. When $\mathrm{m}$ ' or $\mathrm{m}$ approaches zero this would mean energy Ee and A each approach a constant value. With only the field energy Ee and these phenomena one departs from Newtonian gravitation towards GR. Specific for this GR like gravitation is that for $\mathrm{m}>0$ and for $\mathrm{m}=$ 0 there are solutions for the field energy Ee $\sim$ A. $|\mathrm{d}| /|\mathrm{q}|$ with $|\mathrm{d}|=|\mathrm{q}|$ : with $\mathrm{m}$ non zero and Ee space time dependent or alternatively with $\mathrm{m}$ zero and Ee invariant, and the 4 space metric tensor mij space time dependent.

\section{The metric surface area requirement}

The equivalence of the invariant inertial wave energy Es $=n \#$. E and gravitational field energy Ee for a propagation surface can only remain valid when a dimension, different from the propagation surface dimensions, changes during internal energy transfer, for instance radius $|\mathrm{q}|$ or dimension $\mathrm{d}$ or $\Delta 1 \mathrm{t}$, to allow the associated mass $\mathrm{m}$ and momentum $\mathrm{k}_{\text {_ }}$ to change accordingly with this dimension.

When a velocity is non zero and invariant, it relates to invariant coordinate differences or metric distances, ie an invariant non zero space interval. However zero and invariant velocity, relates to zero metric distance, ie an invariant place in space. A Lorentz transformation can transform invariant velocity from non zero to zero or reverse by a change of coordinate system. However with a Lorentz transformation 
metric distances remain invariant. To save covariance, Lorentz transformations, defined with metric surface area invariance instead of and independent of metric distance invariance, can be relevant.

In that case in agreement with paragraph 6. it is not the first order, infinitesimal, approximation of equilibrium derivative transformations, rather the complete, second order, transformations that correspond with Lorentz transformations when these are defined to maintain metric surface area invariance. Lorentz transformations then maintain MV equilibrium time interval $\Delta 1 \mathrm{t}$ change.

\section{Discussion: Radiation waves and star-like localizable or cloud-like non localizable sources}

Several properties can be derived from the above results while the time interval description is maintained in all cases. One of these properties concerns Minkowski 4-space time and gravitation. Another concerns some cosmological estimates. The discussion is included within comments.

\section{Comment I. Surface field energy and surface curvature energy in $n+1$ space time}

For a sphere like 2-surface with an extra dimension d normal to its surface, that is: towards the center of the sphere like surface, there is a reduction of area with a factor $\sim|\mathrm{d}| /|q|$. The dimension $\mathrm{d}$ results in a surface curvature energy linear in $|q|$, indicated with $\operatorname{Ee}(d)$ in the following, alternative to the usual quadratic surface curvature energy [ref. 6]. Introduction of dimension d explains this surface curvature energy to be geometric in origin without any unspecified "curvature" constant. This property connecting geometry and energy includes by itself a theory of gravitation where observable gravitation related phenomena for zero mass or non zero mass waves can be described by geometry. Because of this it is expected that gravitational interference with propagation of non zero mass matter waves and of zero mass radiation waves is similar in geometry.

In essence the construction of field energy $\mathrm{Ee}(\mathrm{d})$ from a surface energy linear in a metric distance is related to the very traditional idea that invariant momentum is maintained by invariant cause: where movement has to be "renewed" or maintained during progress. The Higgs field mechanism where mass of (non zero-) mass acquiring particles has to be created and maintained "often" by energy transfer applies a similar, traditional, and overall space time field approach. The field with energy $\operatorname{Ee}(\mathrm{d})$ is a "not through overall space time" field, however depends on the metric distance related to interval $\Delta 1 \mathrm{t}$ and $\Delta 1 \mathrm{q}$. It is "renewed" or maintained necessarily only within these finite interval domains, not outside these domains. This is an example where in contrast to the traditional radiation wave energy field limit process, paragraph 4 , with a field constructed at every place in infinite one value coordinate space time, the time interval description allows an alternative with a field constructed for specific intervals which can be finite: $\Delta 2 \mathrm{t}$ including $\mathrm{H}$ time dependence, $\Delta 1 \mathrm{t}$ including $\mathrm{H}$ time dependent-like properties, paragraph 7. In [ref. 3] it is derived finite time intervals $\Delta \mathrm{t}$ relate to $\mathrm{H}$ time dependence. The application of the time interval field limit process to construct the field energy Ee, is equivalent to the propagation surface requirement of constant metric surface area A, which can be expressed by theorem c, paragraph 9 .

In the following are assumed 2-surfaces, within Lagrangian equilibrium and including the surface field energy Ee, constructed from $\operatorname{Ee}(d)$ with $|d(n)|=|q(n)|$, in an overall $n+n t$ space time universe. A field on 2surfaces in a 3-space universe, when the space dimension changes in the description of properties, remains a field on 2-surfaces in a n-space universe, for any $\mathrm{n}$. This can be derived from theorem c, discussed in paragraph 9. Then within a overall $n+n t$ space time the surface field energy Ee is the same for all $n$ : 
9. $\quad \mathrm{Ee}=\mathrm{nE} \mathrm{E}|\mathrm{d}(\mathrm{n})| / \mathrm{q}(\mathrm{n}) \mid . \mathrm{A} \sim \mathrm{nE}$. A

E1 is a constant that is determined in [ref. 6] and $|\mathrm{q}(\mathrm{n})|$ is the radial $\mathrm{n}$-space metric distance from qa to $\mathrm{qb}$. The $n$-space normal dimension $d(n)$ is not yet specified, however $|d(n)|$ is assumed to be equal to $|q(n)|$ for all $\mathrm{n}$. A higher dimensional time part with $\mathrm{nt}>1$ is not considered in this paper. In the following a wave propagation surface is assumed to include the normal dimension $\mathrm{d}$ while its time interval description remains valid, from paragraph 7 .

\section{Comment II. Wave propagation surface and time interval $\Delta I t$}

A propagation surface of a radiation or matter wave, emitted by a star source at qb and related to time interval $\Delta 1 \mathrm{t}=[\mathrm{tb}, \mathrm{ta}]$, can be defined as the "same time interval" 2-surface reached during propagation time interval $\Delta 1 \mathrm{t}$. Time interval $\Delta 2 \mathrm{t}$ is a measurement event time interval including wave function collapse. Measurement site qa is located on the propagation sphere for $\Delta 1 \mathrm{t}$ and the line of sight to the star source along $\mathrm{qa}-\mathrm{qb}$ is a radius for the propagation sphere surface.

Surface normal dimension d relates to $\Delta 1 \mathrm{q}$ and assumed is $|\mathrm{d}|=|\Delta 1 \mathrm{q}|=|\mathrm{qb}|$, and with c_light the constant velocity of light and with MV comparative equilibrium $\Delta 1 \mathrm{q}=-\mathrm{q}(\mathrm{t}) / \mathrm{t} . \Delta 1 \mathrm{t}=-\mathrm{c}$ light. $\Delta 1 \mathrm{t}$, and it follows $|\mathrm{d}|$ and $|\Delta 1 \mathrm{q}|$ are both constants for each $\Delta 1 \mathrm{t}$. The character of the propagation surface because of $\mathrm{d}$ means the star source at $q b$ joins the propagation surface since $|\mathrm{d}|=|\mathrm{qb}|$, and qa is a site at the surface by definition, while the radial surface to surface distance is $|\mathrm{qa}-\mathrm{qb}| \approx|\mathrm{qb}|$ when the space origin $\mathrm{q} 0$, with space coordinate value zero, is chosen such that $|\mathrm{qa}-\mathrm{q} 0|<<|\mathrm{qb}|$.

When one localizable source is present, say a star source at qb, wave propagation can be interpreted as a propagation 3-volume from one side at $\mathrm{qb}$ to the other side at the propagation 2-surface. The localizable source and the propagation sphere surface are necessarily included because of the topological difference between sphere surface and sphere volume, ie concerning connectivity qb to qa for all $\Delta 1 \mathrm{t}$. The surface 3 volume is due to the surface normal dimension $d$, not to successive time development of surfaces.

\section{Comment III. Wave propagation energy for time interval $\Delta 1$ t and $n+1$ space time}

Consider time intervals $\Delta 1 \mathrm{t}^{\prime}=\left[\mathrm{t}^{\prime}, \mathrm{ta}\right]$ that vary from $\left[\mathrm{t}^{\prime}=\mathrm{tb}, \mathrm{ta}\right]$ to $\left[\mathrm{t}^{\prime}=\mathrm{ta}, \mathrm{ta}\right]$. Including $\operatorname{Ee}\left(\Delta 1 \mathrm{t}^{\prime}\right)$ for all 2-surfaces for all time intervals $\Delta \mathrm{lt}^{\prime}$ with their metric radius distance $\left|\mathrm{q}^{\prime}\right|=\left|\mathrm{q}^{\prime}\left(\Delta 1 \mathrm{t}^{\prime}\right)\right|$ varying from 0 to $|\mathrm{qb}|$ one derives by integration the complete first order energy EC_e $=\int \mathrm{n} E 1 .\left|\mathrm{d}^{\prime}\right| .1 /\left|\mathrm{q}^{\prime}\right|$. A. d $\left|\mathrm{q}^{\prime}\right|$. This expression includes all energies from all waves emitted and "on the way" during $\Delta 1 \mathrm{t}$. The $\Delta 1 \mathrm{t}$ ' are introduced necessarily because the present discussion remains within the time interval description. Time interval $\Delta 1 \mathrm{t}$ includes wave propagation occurring during all the $\Delta 1 \mathrm{t}$ ' and surface energy for propagation surfaces reached after these $\Delta 1 \mathrm{t}^{\prime}$. Assumed is $\left|\mathrm{d}^{\prime}\right|=\left|\mathrm{q}^{\prime}\right|$ since $\left|\mathrm{d}^{\prime}\right|$ for the $\Delta 1 \mathrm{t}^{\prime}$ propagation surface is limited by the metric radius distance $\mid \mathrm{q}$ ' $\mid$ from the $\Delta 1 \mathrm{t}$ ' surface to the source at qb. Disregarding the metric tensor mij there is $\mathrm{A} \sim\left|\mathrm{q}^{\prime}\right|^{\wedge} 2$ for all $\mathrm{q}^{\prime}$ including $\mathrm{qb}$. Because E_em, the wave emission energy per time unit, is assumed constant, EC_e in equation 10. includes all simultaneous time interval $\Delta 1 \mathrm{t}$ energies Ee. Similarly by integration of the n-surface wave energy Es for distance $\left|\mathrm{q}^{\prime}\right|$ varying from zero to the radius measure for the $\Delta 1 \mathrm{t}$ propagation surface in n-space follows the complete wave energy EC_es with equation 11:

10. $\quad$ EC_e $\sim \mathrm{n} / 3 \mathrm{E} 2 .|\mathrm{qb}|^{\wedge} 3$ 
11. $\quad$ EC_es $\sim$ E3. J Es d $\left|q^{\prime}\right| \sim$ E3. Es. $|\mathrm{qb}| \sim$ E_em. $|\Delta 1 \mathrm{t}|$

The constants E2 and E3 are defined by the integrals in equations 10 and 11 . Every n-surface for all $\Delta 1 \mathrm{t}^{\prime}$ "remains with" the same non localizable surface radiation energy Es independent of the distance $|\mathrm{q}|$ '. EC_e depends on the field surface normal dimension with $|\mathrm{d}|=|\mathrm{qb}|$, just like Ee in equation 9, while EC_es does not, since it depends on wave energy Es.

It is intended that curvature field energy EC_e is the same as propagation wave energy EC_es. This is a valid assertion because of the metric surface area requirement from paragraph 7 .

EC_e part $|q b|^{\wedge} 3$ is independent of the value of $n$ for $n+n t$ space time. These equations can be interpreted as an answer to the question why $\mathrm{n}$ space has three dimensions: one expects the field energy to be proportional to a n-volume. In [ref. 3] arguments are given for a one dimensional time description, among these: time does not admit time reversal. Together with $n$ space with $n$ equals 3 this gives an interpretation for Minkowski space time as 3+1 space time.

\section{Comment IV. Localizable star sources and non localizable cloud sources}

Consider wave emission from a collective mixture of star sources. Any collective propagation surface depends on interference of waves emitted by sources i at different places qi for each i. Recall Newton's mean value requirement for inverse distance ie $1 /|\mathrm{q}|$ dependent energy: at any place within a spherical surface inverse squared attraction from the sphere surface total matter by addition remains a zero sum [Newton, 14].

A cloud collective of similar star sources where the cloud approaches a collective closed surface, not necessarily symmetrical with respect to a space coordinate $q$ within the cloud surface, is origin of a propagation wave surface and wave and field energies Es and Ee, with interaction reduced to zero at q that can be interpreted as wave interference. One can define a fuzzy cloud source collective: star sources i at qi with $|\mathrm{qi}-\mathrm{qb}|<<|\mathrm{qb}|$, with one collective propagation surface and average radius $|\mathrm{q}|=\langle|\mathrm{qi}|\rangle=\langle|\mathrm{qb}+\varepsilon \mathrm{i}|$ $>\approx|\mathrm{qb}|$, and dimensions di with $|\mathrm{di}|=|\mathrm{q} i|$, for all $\mathrm{i}$.

For surfaces with the specific curvature energy, discussed in comment I, the partition function and equation of state was derived in [ref. 6]. A transition temperature Tc exists, proportional to $|q|^{\wedge} 4$, where $|q|$ is the average radius when a set of a variable number of separated surfaces is considered. This set can be reduced to a cloud collective with sources $\mathrm{i}$ and a collective propagation surface. For $\mathrm{T}>\mathrm{Tc}$ the situation is one of many smaller surfaces, for $\mathrm{T}<\mathrm{Tc}$ one of fewer larger surfaces. Now consider only one sphere surface and the average surface radius for the set is a measure for one radius $|q|=|q a-q b|$. The value of radius distance $|\mathrm{q}|$ is decisive for Tc: Tc expected below $\mathrm{T}$ for q "near" q0, and Tc expected above $\mathrm{T}$ for $\mathrm{q}$ "remote from" q0.

When $|\mathrm{q}|$ is interpreted with the correspondence $|\mathrm{q}| \sim|\Delta \mathrm{t}|$ then Tc is proportional to $|\Delta \mathrm{t}|^{\wedge} 4$ and time intervals correspond with properties at distances $|\mathrm{q}|$ : "lower" values for $|\Delta \mathrm{t}|$ and $|\mathrm{q}|$ correspond with a "near" q0 and non localizable fuzzy cloud source collective with reduced wave interaction, "higher" values of $|\Delta \mathrm{t}|$ and $|\mathrm{q}|$ with a "remote from" $\mathrm{q} 0$ localizable star source with measurable wave interaction, values scaled to $T$. This could be of relevance for cosmological space time scales and the emergence of star sources. 
One of the results from paragraph 7. is the description of the emergence of radiation-like waves, "near zero mass" matter waves, at a measurement place qa, "far away" from a localizable source of non zero mass matter waves. This agrees with the $\mathrm{T}<\mathrm{Tc}$ situation "remote from" qa or q0 in both time and space. With $\mathrm{T}>\mathrm{Tc}$ agrees the emergence "near" qa in both time and space, of a non localizable cloud source collective with, when 360 degrees around qa, because of internal wave interference, reduced wave interaction.

\section{Discussion: Cosmological estimates for volume, temperature}

\section{Comment V. Equilibrium theorem c and Liouville's theorem.}

The theorem of continuation of constraints derived in [ref. 6], is inferred in its generalized form, here indicated with theorem $\mathrm{c}$, to have validity to maintain equilibrium within any description including the time interval description. Theorem c seems to represent a situation related to Liouville's theorem concerning phase space discussed for instance in [Arnold, 15], [Goldstein, 16]. The traditional field limit process, paragraph 4 , and the metric surface area requirement equivalent to the field limit process during construction of the surface field energy Ee, paragraph 7, can both be regarded to be a consequence of or equivalent to equilibrium through theorem $\mathrm{c}$ in the time interval description independent of its derivation in [ref. 6]. This and the relation of the time interval description of theorem c and Liouville's theorem is a subject of further study.

Theorem c originally is based on a time moment description derivation. When the generalized theorem $\mathrm{c}$ validates the field limit process this means, while remaining with the same field, phase spaces can be transformed consistently to other phase spaces, within the time interval description, and for instance space time dimension can change as part of the transformation while equilibrium remains maintained. This is applied in paragraph 8. Liouville's theorem applies to phase space in terms of coordinates. The time interval description for theorem $\mathrm{c}$ refers to coordinate interval domains.

\section{Comment VI. Cosmological estimates and overall volume}

When one considers a cosmological overall volume $\mathrm{V}$ that includes a number of sphere surfaces and one assumes the volume is variable, one can apply theorem $\mathrm{c}$ to relate volume $\mathrm{V}$ with the average sphere to sphere distance $|\mathrm{R}|$ to maintain constant density for equilibrium: the distance $|\mathrm{R}|$ remains invariant during equilibrium when $\mathrm{V}$ changes with DV and the number of spheres $\mathrm{N}$ changes accordingly with DN. The interpretation of this cosmology exists in a number of sources for wave emission and their wave propagation surfaces relevant for a time interval $\Delta \mathrm{t}$ like in paragraph 8 . The number of sources or propagation surfaces $\mathrm{N}$ can change when the average radius $|\mathrm{q}|$ changes with $|\mathrm{Dq}|$. The complete sphere surface area is N. A N. $|\mathrm{q}|^{\wedge} 2$, where $A$ is the average metric one-sphere surface area with radius $|\mathrm{q}|$ and where the metric tensor itself is left out. The complete area $\mathrm{N}$. A remains invariant because of the metric surface area requirement, paragraph 7. From $N .|R|^{\wedge} 3=V$ there is $D N .|R|^{\wedge} 3=D V$ and thus $(N+D N) \cdot \mid q+$ $\left.\mathrm{Dq}\right|^{\wedge} 2=\mathrm{N} .|\mathrm{q}|^{\wedge}$. One finds to second order in $\mathrm{Dq} / \mathrm{q}: \mathrm{DV} / \mathrm{V}=-2(\mathrm{Dq} / \mathrm{q})+3(\mathrm{Dq} / \mathrm{q})^{\wedge} 2$ to determine the sign of DV from Dq.

One can describe a cloud collective "near" a space coordinate qb by limiting the above "overall" $\mathrm{N}$ cosmology description towards a collective propagation surface $\mathrm{N}=1$ cosmology. The collective wave propagation surface for $\Delta \mathrm{t}$ is assumed to be one surface, a combination of radii $|\mathrm{qi}|<|\mathrm{q}+\Delta \mathrm{q}|$ for each 
source $\mathrm{i}$ within the variation $\Delta \mathrm{q}$ of the average $|\mathrm{q}|=\langle|\mathrm{q} i|\rangle$, while all other properties follow accordingly. From the discussion for MV comparative equilibrium in the time interval description for $\Delta 1 \mathrm{t}$ there is $|\Delta \mathrm{q}|$ $=\mathrm{c} \_$light. $\Delta \mathrm{t}$, with $|\Delta \mathrm{q}|=|\mathrm{q}|$ leaving out the subscript. One can distinguish several interpretations: one of these is $|\mathrm{qi}|=|\mathrm{qb}+\varepsilon \mathrm{i}|$ and $|\mathrm{q}|=|\mathrm{qb}|$ for a cloud source collective "near" $\mathrm{qb}$.

Writing $|\varepsilon|=<|\varepsilon i|>$ one finds for the cloud source volume $V \_$collective $=q b\left(q b^{\wedge} 2+|\varepsilon|^{\wedge} 2\right)$ where $|\varepsilon|$ is a constant independent of $N$ the number of sources in the collective. Inserted is the assumption $\left\langle|\mathrm{a}|^{\wedge} 2\right\rangle=<$ $|\mathrm{a}|>^{\wedge} 2$ for both $\mathrm{a}=\mathrm{qi}$ and $\mathrm{a}=\varepsilon \mathrm{i}$. For $\mathrm{N}=1$ it follows $|\varepsilon|=\langle|\varepsilon i|\rangle=0$. The 3-volume $\mathrm{V}+\mathrm{DV}$ for $\mathrm{a}$ collective cloud source $>$ the propagation surface 3 -volume $V=|q|^{\wedge} 3=|q b|^{\wedge} 3$ for a localizable star source at the same place $\mathrm{qb}$ in the universe.

One can relate the change V_collective $-\mathrm{V}=\mathrm{DV} \_$collective, from a star source to a cloud source collective with one collective propagation surface, with the above change DV/V for an overall cosmological volume from the limit $\mathrm{N}=1$ to $\mathrm{N}$ otherwise. When $\mathrm{DA}=|\varepsilon|^{\wedge} 2$ and differs from zero this would indicate a non equilibrium collective propagation surface change. When including a constant metric surface area $A$ to maintain equilibrium, the average radius $|q|$ changes with $D|q| \sim|q b|^{\wedge}(1 / 3) .|\varepsilon|^{\wedge}(2 / 3)$.

\section{Comment VII. Cosmological estimates and temperature.}

Consider one surface with metric surface area A, radius $|\mathrm{q}|$, and its sphere volume V_3 A. |q|. Volume $\mathrm{V} \_3$ is assumed to be the cosmological space volume $\mathrm{V}$ since it includes all simultaneous wave or field energy emitted during $\Delta 1 \mathrm{t}$, while $|\Delta 1 \mathrm{t}|$ represents the cosmological time interval scale. Like before the subscript 1 is left out of the following. Thermal volume pressure $\mathrm{P} \sim$ EC_es/V_3 1/A is then assured to be constant within equilibrium by theorem $\mathrm{c}$ and by the metric surface area requirement. The surface field energy EC_e is the same as the wave kinetic energy EC_es, and equal to temperature T: EC_es $\sim|\mathrm{q}| \sim \mathrm{T}$. Assumed is the metric surface requirement for 2-surface area A, from paragraph 7, is valid.

One now applies the ideal gas law for this simple cosmology. The 3+1 space time volume V_4 A. $|\mathrm{q}| \cdot|\Delta \mathrm{t}|$ is proportional to $\mathrm{V} \_3 .|\Delta \mathrm{t}|$, and thus P. V_4 $\sim|\mathrm{q}| \cdot|\Delta \mathrm{t}| \sim \mathrm{T} .|\Delta \mathrm{t}|$, leaving out the constant metric surface area A. Furthermore one assumes $|q|=|\Delta q|$ following the radiation wave generalization, paragraph 5 . The surface space time 4-volume V_4 is proportional to both cosmological volume V_3 and temperature T according to:

\section{2. $\quad$ V_4 $\sim$ A. $|\mathrm{q}| \cdot|\Delta \mathrm{t}| \sim$ A. $|\mathrm{q}|^{\wedge} 2.1 / \mathrm{c} \_$light}

It follows V_4 remains invariant when light velocity c_light remains constant. One finds:

\section{V_4 $\sim$ A. T. $|\Delta t| \sim$ A. T. $|\Delta q| .1 /$ c_light}

This equation is consistent with the discussion of localizable and non localizable sources in paragraph 8 . It now follows from the average radius $|\mathrm{q}|=\langle|\mathrm{qb}+\varepsilon \mathrm{i}|>$ for a one propagation surface cloud source collective:

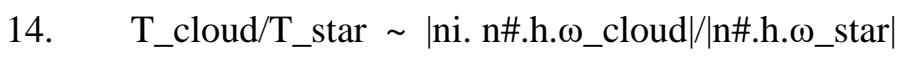

When one inserts for Lagrangian $|\mathrm{L}|=|\mathrm{H}|$ and for $\partial \mathrm{L}=\xi$. $\partial \mu \mathrm{K} \mu$ with the functionals $\xi=1 /|\mathrm{qi}|^{\wedge} 2$ and $\mathrm{K} \mu$ $=|\mathrm{qi}|$ one finds $\partial \mathrm{L}=0$. The $\mathrm{K} \mu$ represent a Noether current with $\partial \mu \mathrm{K} \mu=0$, and the Noether charge 
$\mathrm{Nc}=\int \mathrm{K} \mu$. A. dqi $\sim \mathrm{A}^{\wedge} 2$ while $\mu=4$ and $\mathrm{K} 4=|\Delta \mathrm{t}|$, that however depends on the light velocity c_light to be constant. The infinitesimal $\mathrm{K} \mu$ are equal to the infinitesimal derivative difference transformations, paragraph 6.

From equation 14. one derives < $\mid$ ni $\mid>$ n\#_cloud/n\#_star $\sim$ T_cloud/T_star $~|q|$ star/|q|cloud. When one assumes temperature $\mathrm{T}$ is different for a cloud source or a star source, and the wave momentum and emission energy are different, one finds cloud star source number ni can be derived from measurement: ni.

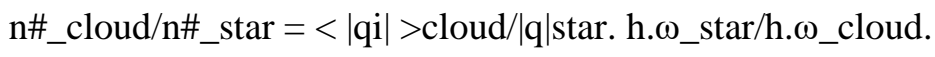

\section{References:}

[1] Hocking, J. G. and G. S. Young: Topology, Addison Wesley (1961)

[2] Einstein, H.: The Foundation of the General Theory of Relativity, in Lorentz, H., Einstein, A., Minkowski, H. and H. Weyl: The Principle of Relativity, reprint of the original publication, Methuen and Company, 1923, translated by Perrett, W. and G. Jeffery, Dover, (1952), from archive.org

[3] Hollestelle, H. H.: Time, Equilibrium, and General Relativity, Int. Jrnl of Science and Technoledge (2020)

[4] Roos, W. J.: Grondslagen van de quantummechanica (Foundations of quantum mechanics), Utrecht University (1978), Giving voluminous matter one value coordinates is justified due to one value coordinates being attributed to its center of weight. Concepts related to a center of weight were applied by Newton in [ref. 14]

[5] Roos, W. J.: ibidem, p. 4: wave function "reduction", otherwise "collapse"

[6] Hollestelle, H. H.: Thermodynamics of a Set of Closed Curved Surface's and Noether's Theorem, Int. Jrnl. of Science and Technoledge (2017)

[7] Sredniawa, B.: Centenary of Five Fundamental Einstein's Papers, Concepts of Physics, Vol. III (2006)

[8] Millikan, R. A.: A Direct Photoelectric Determination of Planck's “h.”, Physical Review, Vol. VII, no. 3, Second series (1916)

[9] Merzbacher, E.: Quantum Mechanics, Wiley (1970)

[10] Beller, M.: Quantum Dialogue, Univ. of Chicago Press (1999)

[11] De Wit, B and J. Smith: Field Theory in Particle Physics, North Holland Physics/Elsevier (1986)

[12] Sakurai, J. J.: Advanced Quantum Mechanics, Addison-Wesley (1978)

[13] Jauch, J. M. and F. Rohrlich: The Theory of Photons and Electrons, Springer (1980)

[14] Newton, I.: Philosophiae Naturalis Principia Mathematica, English edition Andrew Motte Editors (1729), Facsimile reprint Dawsons of Pall Mall (1968), Book I, pg 303, Theorem XXX

[15] Arnold, V. I.: Mathematical Methods of Classical Mechanics (Russian/English translation, K. Vogtmann and A. Weinstein), Springer (1989)

[16] Goldstein, H. B.: Classical Mechanics, Addison Wesley (1978) 